\title{
Nanostructure Research and Amperometric Testing to Determine Detection Capabilities of Biopolymer Matrices Based on Acrylated Epoxidized Soybean Oil
}

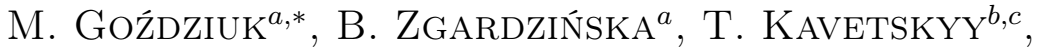 \\ K. Zubrytska ${ }^{b}$, O. SMutoK ${ }^{b, d, e}$, O. ŠAušA ${ }^{f, g}$, \\ M. LeBedevaite ${ }^{h}$, J. OstraUskaite ${ }^{h}$ AND A. KIV ${ }^{i, j}$
}

${ }^{a}$ Institute of Physics, Maria Curie-Sklodowska University,

Pl. M. Curie-Sktodowskiej 5, 20-031 Lublin, Poland

${ }^{b}$ Drohobych Ivan Franko State Pedagogical University,

I. Franko Str. 24, 82100 Drohobych, Ukraine

${ }^{c}$ The John Paul II Catholic University of Lublin, Al. Racławickie 14, 20-950 Lublin, Poland

${ }^{d}$ Institute of Cell Biology, National Academy of Sciences of Ukraine, 79005 Lviv, Ukraine

${ }^{e}$ Department of Chemistry and Biomolecular Science, Clarkson University,

Potsdam, NY 13699-5810 New York, USA

${ }^{f}$ Institute of Physics, Slovak Academy of Sciences,

Dúbravská cesta 9, 84511 Bratislava, Slovakia

${ }^{g}$ Department of Nuclear Chemistry, Faculty of Natural Sciences,

Comenius University in Bratislava, Mlynska dolina, Ilkovicova 6, 84215 Bratislava, Slovakia

${ }^{h}$ Department of Polymer Chemistry and Technology,

Kaunas University of Technology, Radvilenu Rd. 19, 50254 Kaunas, Lithuania

${ }^{i}$ Ben-Gurion University of the Negev, P.O.B. 653, 84105 Beer-Sheva, Israel

${ }^{j}$ South-Ukrainian National Pedagogical University,

Staroportofrankovskaya Str. 26, 65020 Odessa, Ukraine

Doi: 10.12693/APhysPolA.139.432

*e-mail: magdalena.gozdziuk@umcs.pl

\begin{abstract}
The biopolymer matrix synthesized from acrylated epoxidized soybean oil (AESO) and vanillin dimethacrylate (VDM) was examined using the positron annihilation lifetime spectroscopy (PALS) and the amperometry. The matrix version with DMPA (2,2-dimethoxy-2-phenylacetophenone) admixture was also tested in order to check the influence of the admixture on the matrix properties. Samples are intended for the detection of trace pollution with xenobiotics in water. They are carcinogenic substances and have a very negative effect on the human body. The first part of the study examined the nanostructure, and the second tested the matrix for its detection capabilities. The research is aimed at finding the correlation between the nanostructure and the detection properties of the biosensor's biopolymer film.

topics: positron annihilation lifetime spectroscopy, positronium, biosensors, amperometry
\end{abstract}

\section{Introduction}

The development of biotechnology and materials engineering made it possible to synthesize biodegradable and environmentally friendly materials, helping to reduce pollution. One of the types of such materials are biopolymer matrices designed to detect water pollution by xenobiotics, which are a threat to human health, and they are also carcinogenic [1]. The polymer matrix was synthesized on the basis of soybean oil. The positron annihilation lifetime spectroscopy (PALS) was used to determine the size of nanovolumes present in a sample, determine the phase transition temperatures and investigate the sorption/desorption properties of matrices. The PALS is a technique that enables non-invasive testing of samples and provides information about their nanostructure, which is a key issue in determining material properties on the macro scale. The technique is based on the use of the phenomenon of electron $\left(e^{-}\right)$and positron $\left(e^{+}\right)$annihilation.

It is also possible to create an unstable bounded state of an electron and a positron - positronium (Ps), a hydrogen-like atom. In polymers, there is 
a high probability of forming a triplet positronium state - ortho-positronium (o-Ps) [2-4]. The o-Ps lifetime in a medium depends on the size of free volumes (FVs), while the o-Ps intensity correlates with the amount of nanovolumes in the material. We can use the ortho-positron lifetime $\left(\tau_{3}\right)$ determined experimentally to determine the dimensions of the nanovolume using the Tao-Eldrup model $[5,6]$ :

$$
\lambda_{p o}=\frac{1}{\tau_{3}}=2\left[1-\frac{R}{R+\Delta}+\frac{1}{2 \pi} \sin \left(\frac{2 \pi R}{R+\Delta}\right)\right],
$$

where $R$ is the radius of the nanovolume in the biopolymer and $\Delta=0.166 \mathrm{~nm}$ is the empirical constant. The stability of the sample at selected temperatures, the influence of humidity as well as temperature-induced changes of the structure were tested.

The second part of the research was to determine the detection abilities with the use of chronoamperometric analysis, which allows tracking the course of a redox chemical reaction in a solution using current measurements. It is a technique that measures the intensity of the current between a working electrode and an auxiliary electrode in the tested solution. The reference electrode does not polarize when taking measurements. Changes in the current intensity were analyzed depending on the concentration of ABTS in the solution, which was used as a depolarizer (electroactive substance). The obtained chronoamperograms make it possible to track changes of the concentration of the enzyme's substrate at the time in the measuring solution $[7-10]$.

\section{Experimental}

Samples of the biopolymer matrix synthesized from acrylated epoxidized soybean oil (AESO) and vanillin dimethacrylate (VDM) were prepared with photoinitiator 2,2-dimethoxy-2phenylacetophenone (DMPA) and without DMPA, as described earlier [11]. To examine the nanostructure of the sample, the PALS technique was used. The spectrometer was equipped with two Hamamatsu scintillators with detection crystals made of $\mathrm{BaF}_{2}$. The scintillators were positioned at $90^{\circ}$ to each other. The resolution of the measuring system is 240 ps. The sample with the source of positrons was placed in the measuring chamber together with a sample pellets in a "sandwich" arrangement. The ${ }^{22} \mathrm{Na}$ source with an activity of $0.6 \mathrm{MBq}$ was enclosed in Kapton ${ }^{\circledR}$ film with a thickness of $7 \mu \mathrm{m}$.

The measurements consisted of several stages. The first was to check the stability of the sample at $18^{\circ} \mathrm{C}$, and then the temperature was decreased to $-150^{\circ} \mathrm{C}$, where the stability was also tested. The next stage were measurements with increasing the temperature from $-150^{\circ} \mathrm{C}$ to $40^{\circ} \mathrm{C}$ in steps of $10^{\circ} \mathrm{C}$. The measurement for each temperature took $3 \mathrm{~h}$. The aim was to check the influence of temperature on the sample nanostructure and to determine the temperature of the phase transition visible in the change of PALS parameters. Then, analogous measurements with decreasing temperature were performed. The next phase of measurements was to determine the sorption/desorption properties of the sample. In the beginning, steam measurements were made, then liquid water was poured into the chamber, and finally the water was removed from the chamber and the sample was tested under vacuum. The spectra obtained were analyzed using the LT 9.2 program [12]. In this way, information was obtained about the o-Ps lifetime $\tau_{3}$ and intensity $I_{3}$.

In order to investigate the operational characteristics of the bioelectrodes based on AESO/VDM as the enzyme's immobilization matrix, a chronoamperometric analysis was performed. The biosensor configuration included a $\mathrm{Pt}$ counter electrode, an $\mathrm{Ag} / \mathrm{AgCl} / \mathrm{KCl}$ reference electrode, and graphite rods type RW001, $3.05 \mathrm{~mm}$ from Ringsdorff Werke as working electrodes. Before usage, the electrodes were polished with P2000 emery paper Smirdex ${ }^{\circledR}$. The amperometric analysis was carried out using a potentiostat CHI 1200A from IJ Cambria Scientific in an electrochemical cell at $23^{\circ} \mathrm{C}$ under continuous stirring.

The bioelectrodes construction was as follows: dropping of $5 \mu \mathrm{L}$ of laccase solution ( 2 or $10 \mathrm{mg} / \mathrm{mL}$ with an activity $13 \mathrm{U} / \mathrm{mg}$ ) on the working electrode surface and drying for $5 \mathrm{~min}$ at $15^{\circ} \mathrm{C}$; covering the dried enzyme by a thin glue-like layer of AESO/VDM without its additional treatment. To compare the impact of the matrix layer thickness on the efficiency of diffusion in biorecognition layer addition treatment (dissolving) of the AESO/VDM in acetone was done. The $3 \times 3 \times 3 \mathrm{~mm}^{3}$ piece of AESO/VDM matrix was dissolved in $0.3 \mathrm{~mL}$ of acetone. Next, $3 \mu \mathrm{L}$ of the formed polymer-acetone solution was dropped on the enzyme-layer and an ultra thin stable polymer film was formed immediately. The formed bioelectrodes were washed by $50 \mathrm{mM}$ acetic buffer, $\mathrm{pH} 4.5$ and stored at $8^{\circ} \mathrm{C}$ till usage.

\section{Results}

The stability of the samples was tested at room temperature and at $-150^{\circ} \mathrm{C}$. In both temperatures, the PALS parameter remained stable for minimum $20 \mathrm{~h}$ (see Table I). The instability caused by the electron trapping effect [13], which is very

TABLE I

The o-Ps lifetime $\left(\tau_{3}\right)$ and intensity $\left(I_{3}\right)$ at selected temperatures in two investigated samples.

\begin{tabular}{l|c|c|c|c}
\hline \hline \multicolumn{1}{c|}{ PALS parameter } & $\tau_{3}[\mathrm{~ns}]$ & $I_{3}[\%]$ & $\tau_{3}[\mathrm{~ns}]$ & $I_{3}[\%]$ \\
\hline$T$ & \multicolumn{2}{|c|}{$18^{\circ} \mathrm{C}$} & \multicolumn{2}{c}{$-150^{\circ} \mathrm{C}$} \\
\hline AESO/VDM & 2.58 & 26.4 & 1.42 & 24.5 \\
AESO/VDM/DMPA & 2.32 & 26.1 & 1.42 & 23.6
\end{tabular}



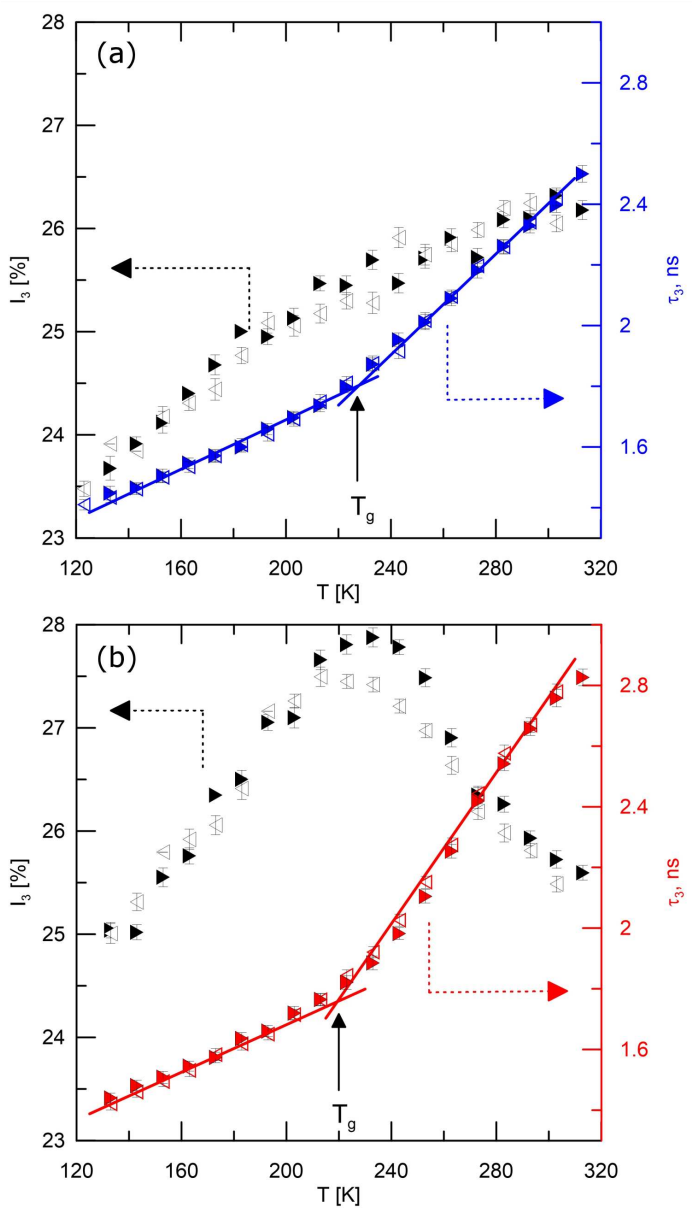

Fig. 1. The o-Ps lifetime $\left(\tau_{3}\right)$ and intensity $\left(I_{3}\right)$ as a function of temperature (empty triangles decreasing temperature, full triangles - increasing temperature) for: (a) AESO/VDM/DMPA and (b) AESO/VDM samples separately.

common among polymers, was not observed here at low temperature. The high stability of the nanostructure of the samples is desirable due to the potential applications of polymers in the production of biosensors.

The AESO/VDM and AESO/VDM/DMPA samples were tested in a wide temperature range from $-150^{\circ} \mathrm{C}(133 \mathrm{~K})$ to $40^{\circ} \mathrm{C}(313 \mathrm{~K})$ (see Fig. 1). In places marked as $T_{g}$, phase transitions occurring in the samples are observed. In the AESO/VDM/DMPA sample, this phenomenon occurs at a slightly higher temperature than in the AESO/VDM sample. Phase transition temperatures are $225.2 \mathrm{~K}$ (increasing temperature) and $220.6 \mathrm{~K}$ (decreasing temperature) in the case of the AESO/VDM sample. The AESO/VDM/DMPA phase transition temperatures are $218.3 \mathrm{~K}$ (increasing temperature) and $224.5 \mathrm{~K}$ (decreasing temperature).

After the phase transition in the AESO/VDM sample, a greater increase in the o-Ps lifetime is visible and it reaches higher values than in $\mathrm{AESO} / \mathrm{VDM} / \mathrm{DMPA}$. In the same sample, higher
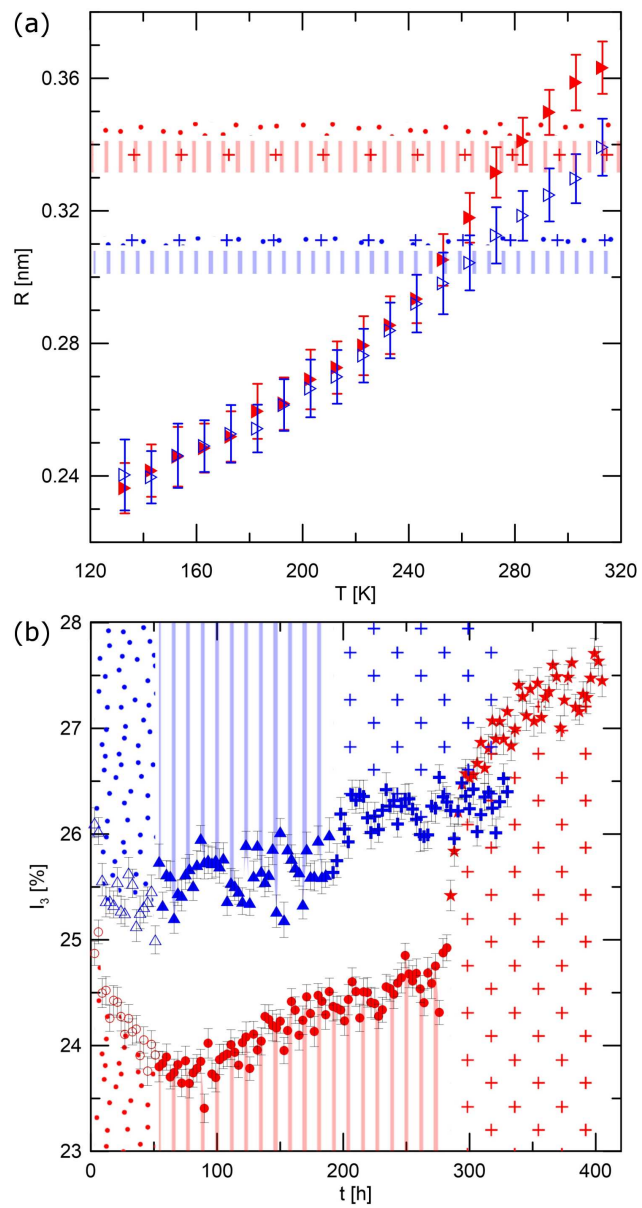

Fig. 2. (a) The radii of free volumes present in the AESO/VDM (full triangles) and AESO/VDM/DMPA (empty triangles) as a function of temperature. (b) The o-Ps intensities under given humidity conditions as a function of time in AESO/VDM (empty dots - vapor $\mathrm{H}_{2} \mathrm{O}$, full points - liquid $\mathrm{H}_{2} \mathrm{O}$, stars - vacuum) and AESO/VDM/DMPA (empty triangles — vapor $\mathrm{H}_{2} \mathrm{O}$, full triangles - liquid $\mathrm{H}_{2} \mathrm{O}$, crosses vacuum). The dotted, hatched, and cross marked areas in both figures correlate separately with the different humidity condition of samples, the vapor $\mathrm{H}_{2} \mathrm{O}$, liquid $\mathrm{H}_{2} \mathrm{O}$ and vacuum, respectively.

values of o-Ps intensities can be observed than in the case of the sample with the DMPA admixture. However, at the moment of a phase transition, these values peak and then begin to decrease. This phenomenon is not observed in the AESO/VDM/DMPA sample, where the o-Ps intensity increases with temperature [14].

On the basis of tests of the samples in a wide range of temperatures, the radii of free volumes in the samples were calculated using the Tao-Eldrup model [5, 6] (Fig. 2a). As the temperature rises, one can observe the increase of the free volume radii in both samples. Below the temperature of $263 \mathrm{~K}$, the radii of free volumes in both samples have similar values, while above this temperature 
in the AESO/VDM sample the radii are bigger than in the case of the AESO/VDM/DMPA sample for the same temperatures. This result suggests that the samples do not differ significantly in their structure below the $T_{g}$, but above this limit temperature, greater free volumes clearly occur in the AESO/VDM sample.

The samples were also tested in the presence of water. In order to test the sorption/desorption properties of the samples, measurements were first performed in the presence of vapor $\mathrm{H}_{2} \mathrm{O}$, then liquid $\mathrm{H}_{2} \mathrm{O}$ was poured, and finally the water was removed from the chamber and the desorption properties of the samples were tested under vacuum conditions. All measurements were performed at the same temperature, which was $291 \mathrm{~K}$. The AESO/VDM sample shows very good sorption properties (Fig. 2b), which is visible in the changes of the o-Ps intensity in the sample in the presence of water. After removing the water from the chamber, the sample does not return to its original state, which is probably due to the permanent bond of water molecules with biopolymer molecules in the sample structure [14]. The AESO/VDM/DMPA sample does not show any sorption properties, which can be seen from slight changes in the o-Ps intensity during tests under given humidity conditions [13]. Under the given humidity conditions, the average o-Ps lifetime remains relatively stable, but a change in the humidity conditions causes changes in the o-Ps lifetime of a continuous nature (in the case of switching from water vapor to liquid $\mathrm{H}_{2} \mathrm{O}$ ) or stepwise (removing water from the chamber).

Based on the o-Ps lifetime, the radii $(R)$ of the free volumes were determined, and the range of their variability in relation to the selected humidity is shown in Fig. 2a. The radii of free volumes in the AESO/VDM sample are higher before adding liquid water (the dotted and dashed area in Fig. 2a, respectively), and do not return to their original value (before the sample was exposed to humidity) after the removal of the water molecules from the chamber (the area with crosses). The situation is different in the AESO/VDM/DMPA sample. The radii of the free volumes are smaller and the presence of water molecules affects the radii of free volumes only when liquid water is present in the sample chamber (the dashed area). Under the conditions of $\mathrm{H}_{2} \mathrm{O}$ vapors and in vacuum, the radius of the free volume is very similar. This means that water is not bound in the sample AESO/VDM/DMPA and can easily be removed from the sample. Therefore, it can be concluded that the addition of DMPA to the sample has a negative effect on its sorption properties.

The AESO/VDM sample, characterized with larger free volumes and showing better sorption properties, was selected for its testing as an effective matrix for laccase immobilization on the electrode surface. For chronoamperometric analysis of AESO/VDM-based bioelectrodes, the working

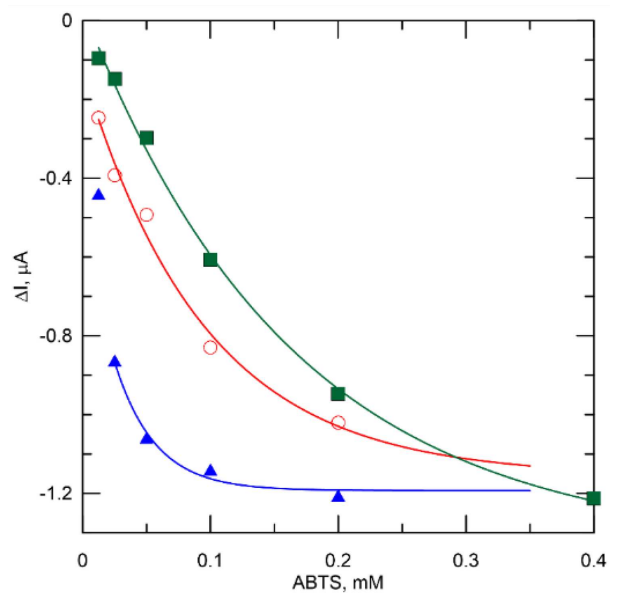

Fig. 3. The current intensity changes $\Delta I$ as a function of ABTS concentration for the AESO/VDM-based bioelectrodes. Symbols: empty dots - untreated matrix with laccase concentration of $10 \mathrm{mg} / \mathrm{mL}$; squares — treated by acetone matrix and $10 \mathrm{mg} / \mathrm{mL}$ enzyme; triangles — treated by acetone matrix and $2 \mathrm{mg} / \mathrm{mL}$ enzyme.

TABLE II

Parameters of exponential curve (2) fitted to experimental curve (Fig. 3).

\begin{tabular}{l|c|c|c|c}
\hline \hline & $\Delta I_{0}$ & $\Delta I_{\max }$ & $\frac{A B T S}{k}$ & $k$ \\
\hline $\begin{array}{l}\text { untreated: } \\
10 \mathrm{mg} / \mathrm{ml} \text { of enzyme }\end{array}$ & -1.156 & 1.032 & 10.5 & 0.095 \\
\hline $\begin{array}{l}\text { treated by acetone: } \\
10 \mathrm{mg} / \mathrm{ml} \text { of enzyme }\end{array}$ & -1.193 & 0.720 & 31.7 & 0.032 \\
\hline $\begin{array}{l}\text { treated by acetone: } \\
2 \mathrm{mg} / \mathrm{ml} \text { of enzyme }\end{array}$ & -1.337 & 1.369 & 6.1 & 0.163
\end{tabular}

potential of $-0.1 \mathrm{~V}$ vs. $\mathrm{Ag} / \mathrm{AgCl}$ was used. This working potential was chosen as the optimal one on the basis of our previous experiments [7]. The calibration curves (intensity changes $\Delta I$ as a function of an increasing concentration of ABTS - the laccase typical substrate) corresponding to chronoamperograms are represented in Fig. 3.

Fitted curves (Fig. 3) with exponential equation allow describing the trend in the change in current:

$$
\Delta I=\Delta I_{0}+\Delta I_{\max }\left(1+\mathrm{e}^{-\mathrm{ABTS} / k}\right),
$$

where $\Delta I_{0}$ is the value down to which $\Delta I$ strives, $\Delta I_{\max }$ is the maximum decrease in current differences observed as a result of the ABTS concentration increasing, and $k$ is a characteristic for the investigated sample constant.

At the first stage of the researches, the bioelectrodes based on untreated AESO/VDM were tested, and then the sample matrix treated by acetone was used to investigate the impact of the matrix layer thickness on the efficiency of diffusion in a biorecognition layer resulting in improving its operational properties $[9,10]$. The parameters of a fitted equation are given in Table II. 


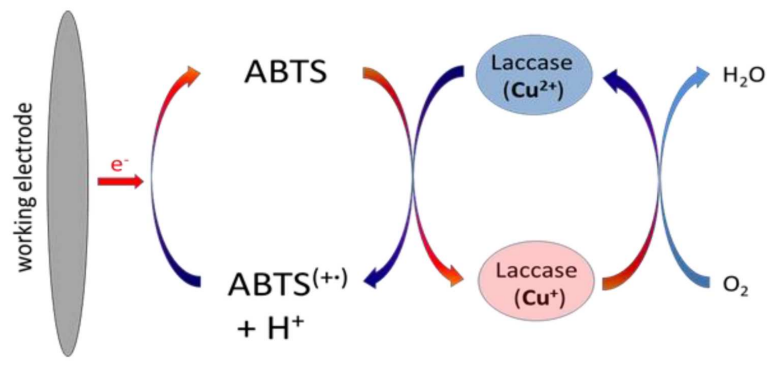

Fig. 4. The electron transfer scheme of the developed laccase-based biosensor.

Samples treated by acetone show different sensitivity (current response) depending on the matrix's diffusion capability and the enzyme concentration. A sample with a high enzyme concentration showed large step changes in current at low ABTS concentrations, but slight changes even with large changes in ABTS concentration. On the other hand, the sample with the enzyme concentration of $2 \mathrm{mg} / \mathrm{mL}$ in the full tested ABTS concentration range reacted with a clearly measurable step change in the current. In the sample untreated by acetone and with the enzyme concentration of $10 \mathrm{mg} / \mathrm{mL}$, the absolute change in the current is the smallest (down to $-3 \mu \mathrm{A}$ ), and the variation in DI is similar to the sample with a low enzyme concentration. This indicates the dependence of the sensor's sensitivity on the matrix thickness resulting in a difference in the diffusion speed. When considering the samples according to the criterion of the clearest response, the best parameters are shown by the sample treated by acetone, $10 \mathrm{mg} / \mathrm{mL}$, for which the constant $k$ is the smallest.

Another point of analysis of the parameters of bioelectrodes based on a different initial concentration of laccase (from 2 to $10 \mathrm{mg} / \mathrm{mL}$ ) is that it is an easier way to get an idea about the principle of biosensor detection: whether it is a biosensor of the "third generation" (direct electron transfer from the electrode surface through laccase to the product) or a mediatorless "first-generation" (based on electron transfer onto an electroactive product of the laccase reaction) biosensor type.

It is a known fact that a direct electron transfer is possible only through the enzyme's monolayer that is directly connected with the electrode surface, all the other enzyme amount does not have any impact on the sensor's output generation. On the other hand, in the case of the "firstgeneration" type biosensor, the electroactive products of enzymatic molecules which are remote from the electrode surface will be affected onto the biosensor's output and the efficiency of this process depends mostly on the matrix diffusion ability. As we can see in Fig. 3, a fivefold decrease of initial enzyme concentration simultaneously significantly decreases the sensor output. This clearly demonstrates that the constructed biosensor relates to the "first-generation" type and the electron transfer scheme between the compounds of the sensor's system is presented in Fig. 4.

Summarizing, it should be noted that the amperometric detection of the developed laccase-based biosensor is based on a reduction of the enzymatically oxidized electroactive product (cation-radicals ABTS $^{+\bullet}$ ) (Fig. 4). The obtained results confirm our previous investigation of the laccase-dependent electron transfer in the biorecognition layer of amperometric biosensors [15].

\section{Conclusion}

The nanostructure study using the PALS method enabled to check the biopolymer matrix stability, observe phase transition temperatures and analyze sorption/desorption properties of the samples. Having obtained data, it was possible to determine the radii of free volumes and to examine the effect of the admixture of DMPA substance on the sample. The nanostructure studies make it possible to check the properties of the sample on the macro scale, and - at the same time - to verify which sample should be tested with the amperometric technique due to its good sorption properties. This sample turned out to be the AESO/VDM matrix.

The use of chronoamperometry made it possible to analyze the detection capabilities of this sample. First, the sample untreated by acetone was tested and the obtained currents were the highest, indicating the poorest detection ability. The acetonetreated samples were characterized by a significant decrease in currents. Thus, it is possible to increase the sensitivity of the bioelectrodes by treating the matrix by acetone resulting in decreasing of a biopolymer film thicknesses and higher diffusion. The detection capabilities of the biopolymer also depend on the enzyme concentration in the matrix. The higher the concentration of the enzyme in the matrix, the greater the detection abilities of the biopolymer film. The combination of different enzyme concentration in the biopolymer matrix allows to establish that the constructed bioelectrodes belong to the first-generation of amperometric biosensors, where easy diffusion of the electroactive substrate has the main impact on the operational parameter of the biosensor.

\section{Acknowledgments}

This work was supported in part by the Ministry of Education and Science of Ukraine (projects Nos. 0118U000297 and 0119U100671), the National Academy of Sciences of Ukraine in the frame of the Scientific-Technical Program "Smart Sensor Devices of a New Generation Based on Modern Materials and Technologies" (project No. 13), the National Research Foundation of Ukraine (project No. 48/02.2020), the Slovak Grant Agency VEGA (project No. 2/0157/17), and the Slovak Research 
and Development Agency (project No. APVV160369). Additionally, T.K. and K.Z. acknowledge the SAIA (the Slovak Academic Information Agency) for scholarships in the IPSAS within the National Scholarship Programme of the Slovak Republic.

\section{References}

[1] F. Ejeian, P. Etedali, H.-A. MansouriTehrani, A. Soozanipour, Ze-Xian Low, M. Asadnia, A. Taheri-Kafrani, A. Razmjou, Biosens. Bioelectron. 118, 66 (2018).

[2] D.M. Schrader, Y.C. Jean, Positron and Positronium Chemistry, 1988.

[3] O.E. Mogensen, Positron Annihilation in Chemistry, Vol. 58, Springer Science \& Business Media, 2012.

[4] Y.C. Jean, Microchem. J. 42, 72 (1990).

[5] S.J. Tao, J. Chem. Phys. 56, 5499 (1972).

[6] M. Eldrup, D. Lightbody, J.N. Sherwood, Chem. Phys. 63, 51 (1981).

[7] T. Kavetskyy, O. Smutok, O. Demkiv, S. Kasetaite, J. Ostrauskaite, H. Švajdlenková, O. Šauša, K. Zubrytska, N. Hoivanovych, M. Gonchar, Eur. Polym. J. 115, 391 (2019).
[8] T. Kavetskyy, O. Smutok, M. Gonchar et al., J. Appl. Polym. Sci. 134, 45278 (2017).

[9] T. Kavetskyy, O. Šauša, K. Čechová et al., Acta Phys. Pol. A 132, 1515 (2017).

[10] T. Kavetskyy, Y. Kukhazh, K. Zubrytska et al., Acta Phys. Pol. A 137, 246 (2020).

[11] M. Lebedevaite, J. Ostrauskaite, E. Skliutas, M. Malinauskas, Polymers. Basel: MDPI AG. 11, 116 (2019).

[12] B. Zgardzińska, T. Hirade, T. Goworek, Chem. Phys. Lett. 446, 309 (2007).

[13] J. Dryzek, J. Kansy, Nucl. Instrum. Methods Phys. Res. A 380, 576 (1996).

[14] M. Goździuk, T. Kavetskyy, K. Zubrytska, et al., "Vegetable Oil-based UV-Cured Polymers: I. Phase Transition and Adsorption Properties Probed by Positron Annihilation", submitted to ACS Appl. Mater. Interfaces (2020).

[15] T. Kavetskyy, O. Smutok, O. Demkiv et al., Mater. Sci. Eng. C 109, 110570 (2020). 